\title{
Design and Application of a Free and Lightweight Aquaculture Water Quality Detection Robot
}

\author{
Liwen Huang, Zhiwen Li, Sirui Li, Li Liu, Yinggang Shi* \\ College of Mechanical and Electronic Engineering, Northwest A\&F University, Yangling 712100, China
}

Corresponding Author Email: syg9696@nwsuaf.edu.cn

https://doi.org/10.18280/jesa.530114

Received: 10 October 2019

Accepted: 17 December 2019

\section{Keywords:}

freshwater aquaculture, water quality detection, underwater robot, three-propeller propulsion, control system, remote monitoring

\begin{abstract}
Currently, the water quality detection of freshwater aquaculture environment is generally completed by humans; however, manual testing can only detect the surface water quality, and the testing job is a kind of high-intensity and high-risk operation that has to be done on the broad aquaculture waters under burning sun, strong wind, or cold weather conditions. Targeting at these problems, this study designed a robot for the aquaculture water quality testing job. The robot uses an equipped water quality detection module to collect parameters such as the water temperature, $\mathrm{pH}$ value, and dissolved oxygen (DO) content of the freshwater aquaculture environment from fixed points in real time. Moreover, the designed robot has an open-frame structure that adopts a three-propeller propulsion mode, two propellers provide vectorial-force propulsion and one propeller provides fixed-force propulsion for the robot, so that it could achieve free turning and has a light weight. At last, the study designed the control system of the robot and tested the performance of a prototype, the test results showed that, in terms of navigation control, dive control, water quality detection, and water parameter collection, the robot had satisfied the working requirements.
\end{abstract}

\section{INTRODUCTION}

China has the largest aquaculture scale in the world [1-4]. At present, aquaculture in China is dominated by closed water environments such as ponds and cages. Freshwater aquaculture [5] generally uses ponds, reservoirs, rivers, lakes and other freshwater bodies to farm fishes, shrimps, crabs, shellfishes, lotuses, and lotus roots, etc. The water quality of the freshwater aquaculture environment affects the output, aquatic product quality and economic benefits of the aquaculture industry [6]; and the key parameters that can affect aquaculture [5-7] include: water temperature (T), $\mathrm{pH}, \mathrm{DO}$, electrical conductivity (EC), redox potential, turbidity, light, ammonia nitrogen, and sulfide, etc. Now, the culture depth of common freshwater fish ponds is usually about 4 to 5 meters [8], and fishes generally move within 2 to 3 meters of water depth. In terms of water quality detection, most domestic farmers still adopt the manual sampling method, which is not real-time, and it is quite time and labor consuming. Manual operation has the characteristics of limited depth, narrow operating range, high intensity, and high risks, which have severely affected the development of aquaculture industry. Some major aquaculture enterprises have installed online water quality monitoring equipment in their aquafarm, but the equipment is generally installed in fixed positions. Considering the diversity and variability of the breeding environments, in order to obtain more comprehensive information, it's often required to install multiple monitoring equipment in more positions, which would greatly increase the procurement and maintenance costs [9]. In recent years, some foreign countries have applied water quality detection robots to the aquaculture industry and achieved remarkable results; and domestic research has also begun to develop underwater robots for the aquaculture industry, but our research in this field is basically in its infancy [10-12].

Water quality directly affects the growth of aquatic animals and the quality of aquatic products. Therefore, water quality monitoring and early warning are the most important parts of aquaculture management [13]. Due to the openness feature of the aquaculture environment, the water quality parameters are often non-linear, dynamic, variable, and complex. At present, water quality prediction and early warning are mainly for parameters such as DO, water temperature, and $\mathrm{pH}$ [14]. Considering that temperature and DO are the main factors affecting fish feeding, these two parameters are often taken as indicators for the feeding control of freshwater fish farming, and gradually becoming one of the feeding management decisions [15]. For example, Cheng et al. [16] carried out experiments to investigate the response of fish growth to the water environment and predict the growth rate of fish under the multi-factor synergy of the aquaculture water environment, and the results showed that: within a certain range, with the increase of DO content, both the fish food conversion efficiency and the specific growth rate of fish have increased as well. Zhou et al. [17] used near-infrared imaging technology to observe the fish feeding process, and provided a new method for quantitatively studying the changes in fish feeding behavior, indicating that the study of fish feeding behavior [18] is of positive significance for fish feed releasing, which can effectively reduce water pollution, improve the well-being of aquatic animals, thereby the feeding decisions could be made based on the behavior of the fish or related parameters.

In freshwater aquaculture, the underwater operating environment is very complicated, which has placed new 
requirements on the flexibility of the water quality detection robot. To this end, this study designed a water quality detection robot (hereinafter referred to as "the robot") for aquaculture water quality detection. The propelling direction of the vectorial-force propulsion device of the robot is changeable, and the robot has the characteristics of compact structure and flexible movement, it can be equipped with water quality detection module to achieve large-scale underwater environment detection. The remote monitoring terminal receives the data sent by the robot, visualizes the data through detection software and displays it on the host computer. The robot operator only needs to stand on the shore to quickly and easily detect the water quality and collect the three parameters of water temperature, $\mathrm{pH}$, and $\mathrm{DO}$ from fixed points in realtime.

\section{MATERIALS AND METHODS}

To solve the problems of slow speed, complex operation, high intensity, small detection range, and large difficulty in manual water quality testing, this study designed a water quality detection robot suitable for common aquaculture fish ponds, by analyzing the aquaculture environment, the performance parameters of the robot were designed as shown in Table 1. The maximum dive depth is $6 \mathrm{~m}$, the speed is less than $2 \mathrm{Kn}$, and the underwater endurance is 2 hours. The robot can replace humans to perform underwater operations and complete comprehensive and efficient water quality detection tasks in target water areas.

Table 1. Performance parameters of water quality detection robot

\begin{tabular}{ccccccc}
\hline Parameter & Size & Weight & $\begin{array}{c}\text { Number of } \\
\text { propellers }\end{array}$ & Navigation & Internal testing & $\begin{array}{c}\text { External detection } \\
\text { parameter }\end{array}$ \\
\hline Indicators & $1 \times 0.5 \times 0.35 \mathrm{~m}$ & $\leq 15 \mathrm{~kg}$ & 3 & BDS/INS & Temperature, Humidity & $\begin{array}{c}\text { Depth, Water temperature, } \\
\text { pH value, Dissolved oxygen }\end{array}$ \\
\hline
\end{tabular}

\subsection{Mechanical system}

\subsubsection{Overall layout}

Considering the water quality detection function of the robot, the overall structural layout of the robot was designed as shown in Figure 1. The structure includes the casing, pressure compartment, propulsion device, control system, etc. The robot adopts a frame structure, the casing includes the side plates and the main frame, the side plates are firmly connected with the main frame or the casing is integrally formed as a whole. The front and rear bezels are made of transparent fiberglass, which facilitates subsequent system upgrades and carrying the vision sensor. The robot adopts a three-propeller propulsion mode (with two propellers provide vectorial-force propulsion and one propeller provides fixed-force propulsion) to make the robot perform movements such as moving forward and backward, diving, and yawing, etc. The robot has two rectangular cuboid pressure compartments, one for batteries and one for electronic devices. The battery compartment is for installing the power module, and the electronic device compartment is for installing the components of the control system circuit and the water quality detection sensors; the BDS module and the inertial components together act as the navigator of the robot. At the same time, a temperaturehumidity sensor is installed in the compartment to detect whether there's water in the pressure compartment and give early warning of the leak if necessary, so as to ensure the normal operation of the robot.

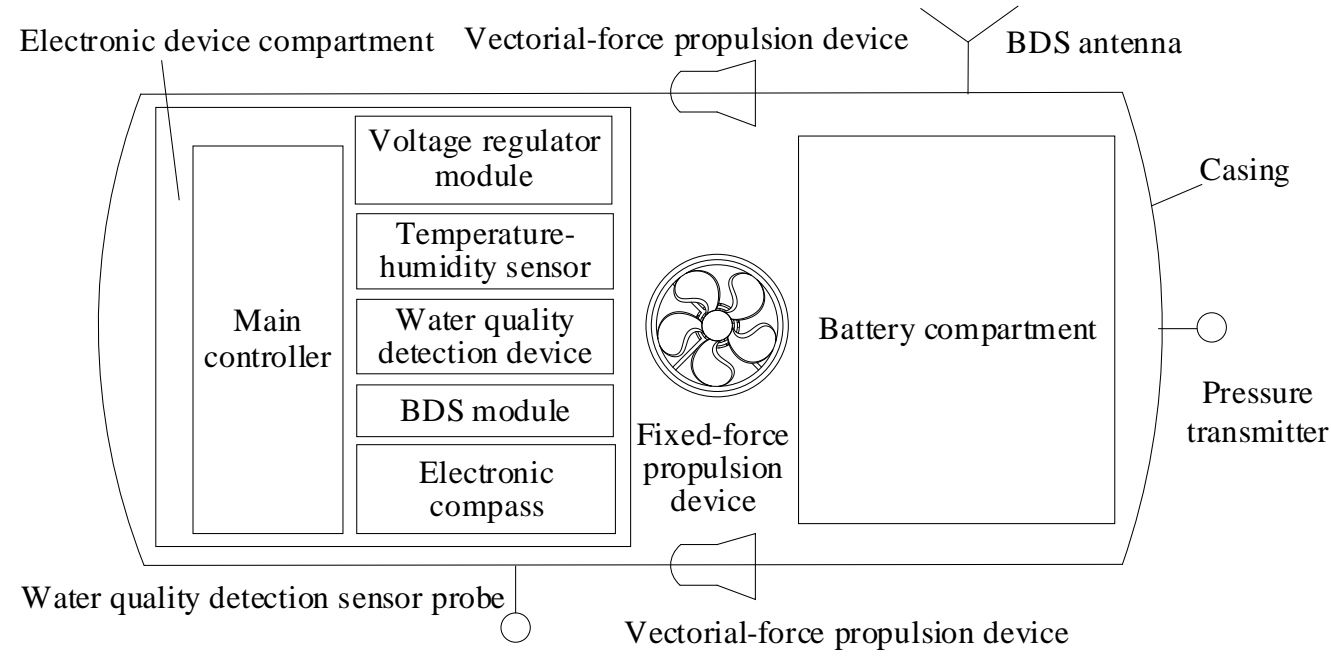

Figure 1. Overall structural layout of the robot

Ordinary water quality detection robots generally adopt the propeller and water-jet propulsion system, the robots have a low speed and are sensitive to the costs. The propeller propulsion system has a simple structure and a low cost, therefore, it's the first choice for water quality detection robots. The robots need to perform movements of $\mathrm{N}$ degree of freedom, so $\mathrm{N}$ propulsion devices are required at least [19]
The robots need to perform moving forward and backward, diving and floating (moving up and down), yawing, and other movements, theoretically, two vectorial-force propulsion devices could do the work, but in order to reduce the cost of the control system and facilitate the operation of the robot, two vectorial-force propulsion devices were adopted to help the robot perform the moving forward/backward and yawing 
movements, and the fixed-force propulsion device was adopted to help the robot perform diving and floating (moving up and down).

To enhance the control flexibility of the robot, the layout of the propulsion devices of the proposed robot arranged the two vectorial-force propulsion devices horizontally, and the fixedforce propulsion device vertically, as shown in Figure 2. When arranging the positions of the propellers, the following 3 principles were taken into consideration [20]:

(1) To eliminate the additional movements of the robot, the combined force of the propellers must intersect at the gravity center of the robot;

(2) To reduce the vibration of the robot, the reaction force of the propellers to the robot needs to be decreased;

(3) To improve the propulsion efficiency, the positions of propellers should be as parallel as possible to the moving direction of the robot.

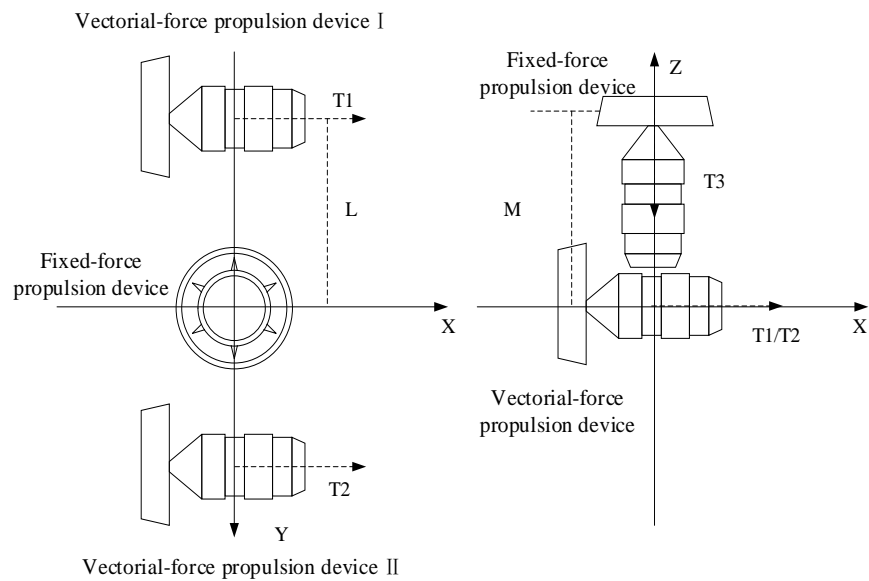

Figure 2. Propulsion device position

Assume the combined thrust force and combined torque received by the robot are $T$ and $M$, respectively; the thrust generated by the propulsion devices and the torque of the gravity center are $\sum_{1}^{3} T_{i}$ and $\sum_{1}^{3} M_{i}$, respectively; in this layout, the thrust and torque generated by the propulsion devices are:

$$
\left[\begin{array}{c}
T \\
M
\end{array}\right]=\left[\begin{array}{l}
\sum_{1}^{3} T_{i} \\
\sum_{1}^{3} M_{i}
\end{array}\right]=\left[\begin{array}{c}
T_{1}+T_{2} \\
0 \\
T_{3} \\
0 \\
0 \\
L \times\left(T_{1}+T_{2}\right)
\end{array}\right]
$$

This scheme only needs three propellers to generate driving forces for six degrees of freedom, and there is no mutual coupling between each degree of freedom [21]. Since the robot doesn't rely on the tail rudder for steering, it is very flexible in turning directions and is capable of completing detection tasks in complex environments.

\subsubsection{Robot frame}

The frame of robot provides a support and protection for the installation of the components and parts of the robot. To meet mechanical strength, processability and assembly requirements of water quality detection robots, and achieve low density and low water absorption features, ABS and
PMMA had been selected as the materials for the frame of the robot, as shown in Figure 3, the designed structure includes: front bezel, back bezel, side bezel, side bracket, battery compartment support plate, electronic device compartment support plate, dive/float motor fixation plate, buoyancy block fixation plate and other structures. Wherein, the side bezel, side bracket, battery compartment support plate, electronic device compartment support plate, dive/float motor fixation plate, and buoyancy block fixation plate are made of lowdensity ABS; while the front and rear bezels are made of transparent PMMA, which is convenient for carrying various sensors in subsequent studies.

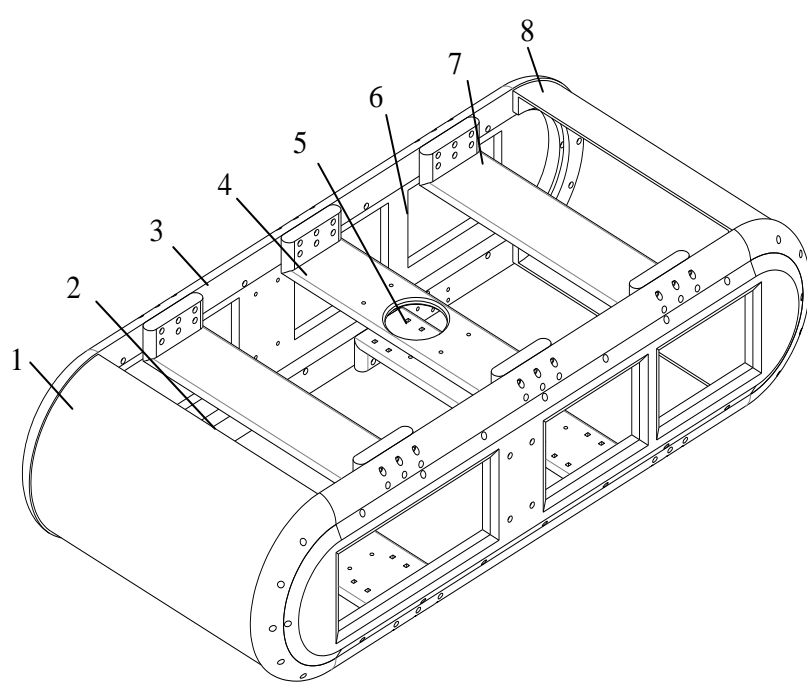

1. Front bezel 2. Battery compartment support plate 3 . Side bracket 4 Dive/float motor fixation plate 5. Electronic device compartment support plate 6 . Side bezel 7. Buoyancy block fixation plate 8 . Rear bezel

Figure 3. Robot frame

\subsubsection{Propulsion device}

The vectorial-force propulsion device provides the thrust for the robot's movements such as forward, yaw, pitch, and roll, and the direction of the thrust is changeable, the designed mechanical structure of the vectorial-force propulsion device is shown in Figure 4. The brushless motor is installed in the motor compartment, the motor shaft protrudes from the motor compartment and connects to the propeller shaft through a universal joint coupling to drive the propeller to rotate. The spatial linkage system is composed of a crank and connecting rods, it drives the propeller through two waterproof steering gears to achieve vertical and horizontal movements of the propeller. When the propeller moves in the vertical direction, the steering gear 1 drives the crank to rotate, then through the short connecting rod, it drives the cross linker to rotate around the fixed hinge of the motor compartment. When the propeller moves in the horizontal direction, the steering gear 2 drives the crank to rotate, then through the long connecting rod, it drives the propeller frame to rotate as well.

The fixed-force propulsion device provides thrust for the robot's movements of dive and float, its structure is shown in Figure 5, including a motor compartment, a brushless motor, a coupling, a propeller shaft, a shroud, and a propeller. The brushless motor in the motor compartment is connected to the propeller through a coupling to drive the propeller to rotate, generate thrust, and push the robot to dive. 


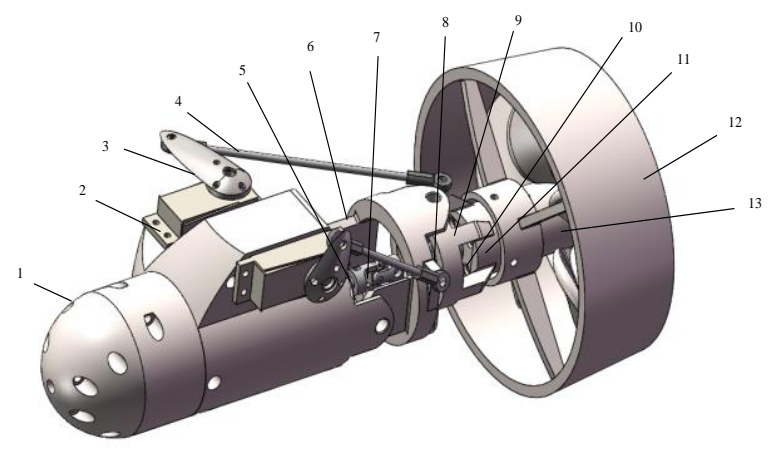

1. Motor compartment 2. Waterproof steering gear 3. Crank 4. Connecting rod 5. Brushless motor 6 . Cross linker 7. Universal joint coupling 8. Propeller shaft 9. Bearing end cover 10. Cylindrical roller bearing 11 . Propeller frame 12. Shroud 13. Propeller

Figure 4. Structure of vectorial-force propulsion device

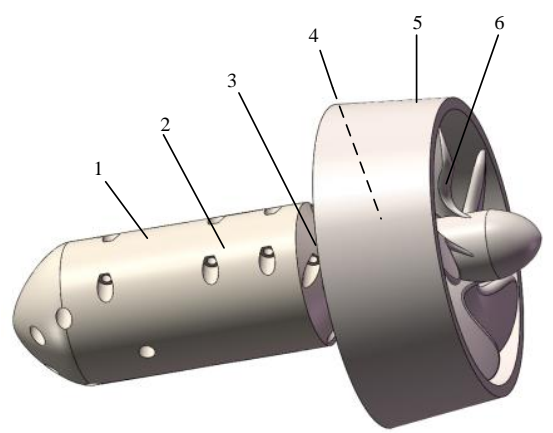

1. Motor compartment 2. Brushless motor 3. Coupling 4. Propeller shaft 5. Shroud 6. Propeller

Figure 5. Structure of fixed-force propulsion device

The cross-sectional area (CSA) of the robot was $A=$ $1 \times 0.5 \times 0.35=0.175 \mathrm{~m}^{2}$, the water density of the breeding water was $\rho=1 \mathrm{~kg} / \mathrm{m}^{3}$, the sailing speed of the robot was $v=$ $0.7 \mathrm{~m} / \mathrm{s}$, the dimensionless drag coefficient was $C_{d}=2$, then the sailing resistance of the robot was:

$$
F_{N}=\frac{1}{2} \rho v^{2} A C_{d}=85.75 \mathrm{~N}
$$

The robot is driven by a DC brushless motor and is affected by the resistance of the water flow during navigation. The thrust generated by the propulsion devices mainly applies the work to overcome the navigation resistance. The propulsion efficiency of the motor took $\eta_{1}=0.95 \sim 0.98$, and the propulsion efficiency of the propellers took $\eta_{2}=0.45$, then the propulsion efficiency of a single propeller was:

$$
P_{\text {propulsion }}=\frac{1}{2} F_{N} v=30.01 \mathrm{w}
$$

The power required by a single propulsion motor was:

$$
P_{\mathrm{m}}=\frac{F_{\text {propulsion }}}{\eta_{1} \eta_{2}}=70.2 \mathrm{w}
$$

Therefore, a brushless motor (Model: 42BL80S09-230, manufactured by Beijing Time Chaoqun Electrical Technology co. LTD, Beijing, China) with a rated power of
$90 \mathrm{~W}$ was selected for the proposed robot. Its performance parameters are shown in Table 2.

Table 2. Performance parameters of Model 42BL80S09-230 brushless motor

\begin{tabular}{cccccc}
\hline $\begin{array}{c}\text { Rated } \\
\text { voltage }\end{array}$ & $\begin{array}{c}\text { Rated } \\
\text { power }\end{array}$ & $\begin{array}{c}\text { Rated } \\
\text { torque }\end{array}$ & $\begin{array}{c}\text { Rated } \\
\text { speed }\end{array}$ & $\begin{array}{c}\text { Rated } \\
\text { current }\end{array}$ & Size \\
\hline $24 \mathrm{~V}$ & $90 \mathrm{~W}$ & $\begin{array}{c}0.3 \\
\mathrm{~N} \cdot \mathrm{m}\end{array}$ & $3000 \mathrm{r} / \mathrm{min}$ & $4.7 \mathrm{~A}$ & $\begin{array}{c}42 \times 42 \times 80 \\
\mathrm{~mm}\end{array}$ \\
\hline
\end{tabular}

The propeller outer diameter was $D=120 \mathrm{~mm}$, the pitch $\mathrm{P}=120 \mathrm{~mm}$, the rotation speed $N=80 \mathrm{r} / \mathrm{min}$, then the output power of the propeller was:

$$
W=\left(\frac{D}{10}\right)^{4} \times\left(\frac{P}{10}\right) \times\left(\frac{N}{1000}\right)^{3} \times 0.45=57.33 W>P_{\text {propulsion }}=30.01 \mathrm{w}
$$

The output power of the propeller met the power requirements.

\subsubsection{Pressure compartment}

The pressure compartment provides a water-free environment for the battery and electronic components. In view of the robot's working requirements and processing costs, the pressure compartment was made of ABS material, as shown in Figure 6. The casing of the pressure compartment and the end cover are connected by flange. The connector interface on the side of the support frame is convenient for connection of various electronic components. The ceiling and bottom plates of the support frame are used to fix the battery and various electronic components, they are connected to two support plates by rotating hinges, forming a movable structure. When the support frame is pushed into the casing of the pressure compartment, as the rotating hinge is pushed up, the support plates are put up and closely clung to the side wall of the casing of the pressure compartment. In the process of realizing automatic casing-wall clinging, the contact area between the components and the inner wall of the casing was increased to achieve efficient heat dissipation.

The connection position between the electrical components in the pressure compartment and the external circuit needs to be well sealed, so sealing grooves were set at the connection position and double $\mathrm{O}$-shaped sealing rings were installed to prevent leakage and reinforce the seal, so that when the robot is operating in turbid or sandy water areas, water won't enter the pressure compartment.

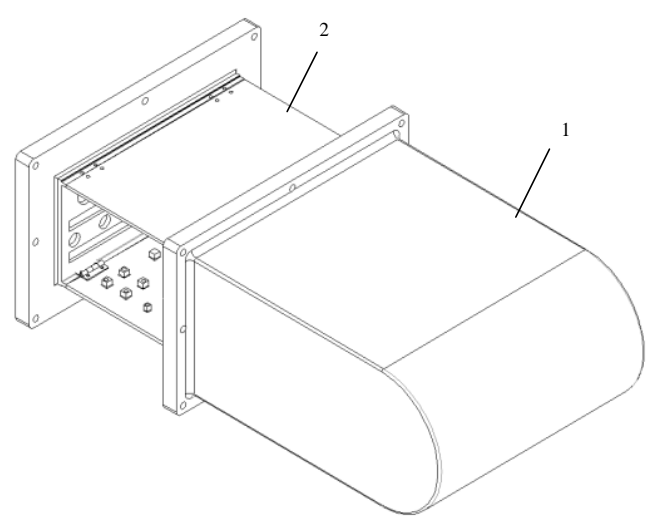

1.Pressure compartment casing 2. Support frame

Figure 6. Pressure compartment structure 
115

The areas of the ceiling and bottom plates of the pressure compartment casing were the largest, the possibility of failure was the largest, the casing wall thickness was designed according to the size of the areas of the ceiling and bottom plates, the medium density was $\rho=1 \mathrm{~kg} / \mathrm{m}^{3}$, the gravitational acceleration $g=9.8 \mathrm{~m} / \mathrm{s}^{2}$, maximum dive depth of the robot was $h={ }_{\max }$; according to the elastic mechanical strength design criteria, the maximum pressure the robot has to bear when diving was:

$$
P_{\max }=\rho g h_{\max }=5.88 \times 10^{-2} \mathrm{MPa}
$$

The pressure compartment material is polypropylene, yield limit $\sigma_{b}=65 \mathrm{MPa}$, allowable yield limit $\left[\sigma_{s}\right]=0.6 \sigma_{b}=$ $39 M P a$, safety factor $K=2.5$, height of the side plate of the casing $b=200 \mathrm{~mm}$, maximum stress coefficient $\beta=0.272$, then minimum wall thickness of the pressure compartment casing was:

$$
t \geq K \sqrt{\frac{\beta P_{\max } b^{2}}{\left[\sigma_{s}\right]}}=10.2 \mathrm{~mm}
$$

Considering the size of the threaded hole, the pressure compartment wall thickness was selected to be $t=15 \mathrm{~mm}$.

If the mass of each component was $M_{i}$, then the total mass was $M=\sum_{i=1}^{n} M_{i}$; if the buoyancy of each component was $F_{i}$, then the total buoyancy was $F=\sum_{i=1}^{n} F_{i}$. The coordinate of the equivalent center of gravity of the robot was $\left(X_{g}, Y_{g}, Z_{g}\right)$, the coordinate of the mass center of each component was $\left(x_{i}, y_{i}, z_{i}\right)$, the coordinate of the equivalent buoyancy center of the robot was $\left(X_{f}, Y_{f}, Z_{f}\right)$, the coordinate of the buoyancy center of each component was $\left(X_{i}, Y_{i}, Z_{i}\right)$, then the robot's equivalent center of gravity and equivalent center of buoyancy could be calculated, order the coordinate of the robot's equivalent center of buoyancy to minus the coordinate of its equivalent center of gravity, then there are:

$$
\begin{aligned}
& X_{f}-X_{g}=\frac{\sum_{i=1}^{n} F_{i} X_{i}}{F}-\frac{\sum_{i=1}^{n} M_{i} x_{i}}{M}=29.65-30.95=-4.3 \mathrm{~mm} \\
& Y_{f}-Y_{g}=\frac{\sum_{i=1}^{n} F_{i} Y_{i}}{F}-\frac{\sum_{i=1}^{n} M_{i} y_{i}}{M}=0-0=0 \mathrm{~mm} \\
& Z_{f}-Z_{g}=\frac{\sum_{i=1}^{n} F_{i} Z_{i}}{F}-\frac{\sum_{i=1}^{n} M_{i} z_{i}}{M}=118.29-80.63=37.66 \mathrm{~mm}
\end{aligned}
$$

It can be known from Formula (8) that the positions of the center of gravity and the center of buoyancy of the robot were not far from each other. By adding clump weights and floatation blocks, the force acting on the robot during underwater activities could be balanced and its movements could be stabilized.

The components made of ABS material were firmly installed on the open frame of the robot by bolting. The structure could resist the impact of fishes, shrimps and crabs on the robot without affecting its movement in the water. After assembly, the robot structure is as shown in Figure 7, the robot was $1 \mathrm{~m}$ long, $0.5 \mathrm{~m}$ wide, and $0.25 \mathrm{~m}$ high, the steering and moving were quite flexible.

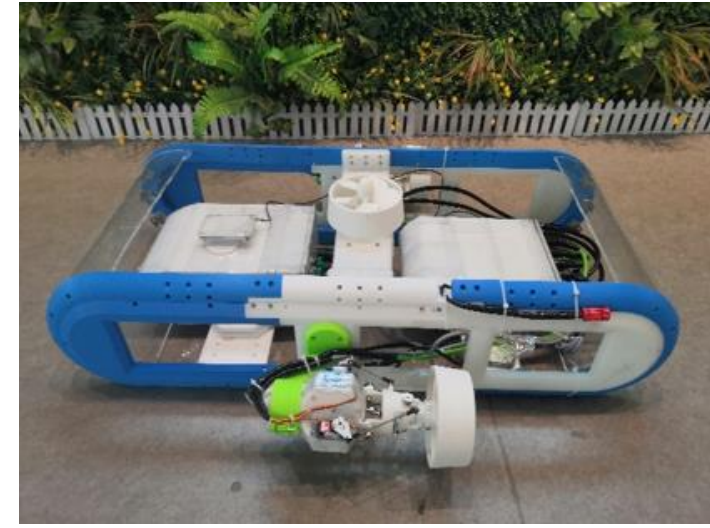

Figure 7. A picture of the robot

\subsection{Control system}

\subsubsection{Control system structure}

The control system of the robot adopted a distributed structure, as shown in Figure 8. It mainly includes a wireless communication module, a power module, a water quality detection module, a depth keeping control module, a positioning control module, a motor drive module, a steering gear control module, and a main controller. Wherein, the wireless communication module uses wireless serial port for communication; the power module supplies power for other modules; when the power supply module works, the robot switch is turned on, the main controller starts to work and controls the relay; the main controller controls all modules; the water quality detection module contains a water quality detection sensor and a water quality control module; in the depth keeping control module, the pressure transmitter communicates with the main controller through the signal conversion module; the positioning control module consists a BDS module and an electronic compass, which can achieve surface and underwater navigation and positioning control respectively; in the motor drive module, three motor drivers are connected to the main controller to control the operation of the motor; the steering gear control module controls four steering gears through four steering gear controllers, so as to achieve the space vector motions of the propellers.

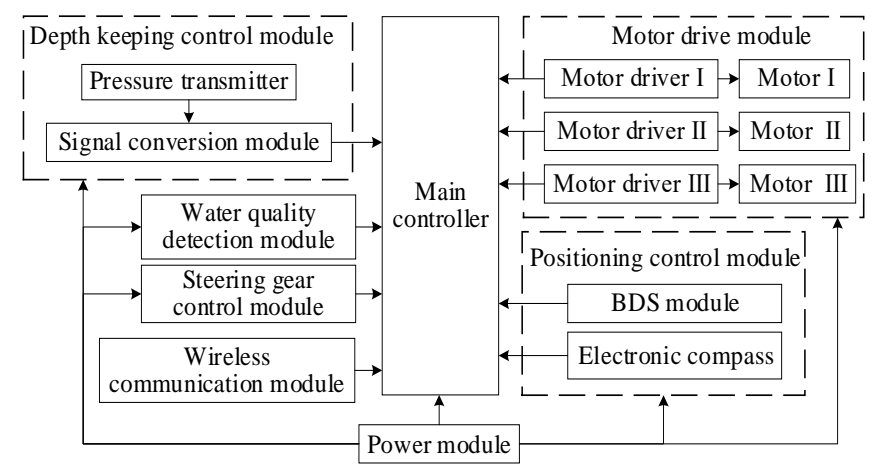

Figure 8. Overall structure layout of the control system

In order to control the vector motion of the propulsion device, $180^{\circ}$ digital waterproof steering gear (model DS3218, manufactured by Dongguan Dasheng Steering Gear Technology co. LTD, Dongguan, Guangdong, China) and steering gear driver (model LSC-16, manufactured by Shenzhen Le Huan sol Technology co. LTD, Shenzhen, Guangdong, China) were selected. Temperature and humidity 
sensor (model AM2305, manufactured by Guangzhou Lehang Electronic co. LTD, Guangzhou, Guangdong, China) with high sensitivity and high reliability was selected for the early warning of pressure compartment leakage. The navigation of the robot contained two parts: a navigation module (model ATK1218-BD, manufactured by Guangzhou Xingyi Electronic Technology co. LTD, Guangzhou, Guangdong, China) for surface navigation, and an electronic compass (model JY901, manufactured by Shenzhen Witt Intelligent Technology co. LTD, Shenzhen, Guangdong, China) for underwater navigation. When the robot is diving, it uses the pressure transmitter (model ELE-801, manufactured by Eleco Electric co. LTD, Yueqing, Zhejiang, China) to measure the pressure, and convert the pressure value into the robot's dive depth and sends it to the main controller.

\subsubsection{Water quality detection module}

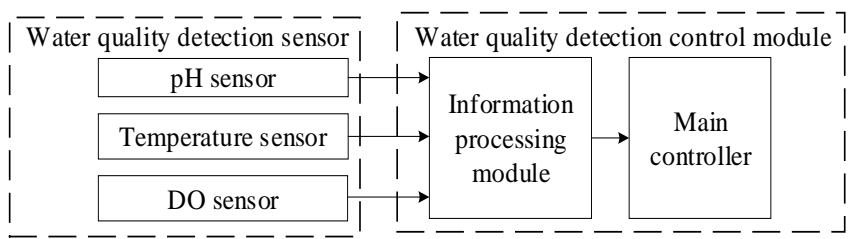

Figure 9. Structure of water quality detection module

In order to detect the temperature, $\mathrm{pH}$ and $\mathrm{DO}$ of the water area, a detection system shown as Figure 9 had been designed in this study, wherein a $\mathrm{pH}$-temperature transmitter (model D144, manufactured by Rirans electronic technology co. LTD, Shenzhen, Guangdong, China) was adopted as the temperature sensor and the $\mathrm{pH}$ sensor, and a LDO (Luminescence measure
For Dissolve Oxygen) (model YDSBS-3001-TT, manufactured by Weihai jingxun unimpeded electronic technology co. LTD, Weihai, Shandong, China) sensor was adopted as the DO sensor.

\subsubsection{Power module}

The power supply system is the core part of the robot. It provides energy support for the safe operation of the robot, it supports remote on/off control, and has the function of leak warning. In this study, the power system mainly includes: charging ports, power switch module, leakage warning module and overload protection module. The charging ports use the waterproof connector of the robot to realize the connection between the power supply and the charger. The working principle of the power supply system is shown in Figure 10.

When there's leak in the electronic device compartment, the leak warning signal issued from the electronic device compartment will be received by the power module, then the leak warning module starts to work and cuts off the power output circuit; when there's short circuit fault in the electronic device compartment, the overload protection module starts to work and the overload protector cuts off the power to protect the circuit of the electronic device compartment.

Once there's leak in the pressure compartment, the leakage early warning module can cut off the power in time, meanwhile the wireless communication module sends the information of the last position provided by the navigation module to the remote monitoring terminal on the host computer, so that the robot could be salvaged later. During subsequent system upgrades, targeting at the problem of the difficulty in robot retrieve after power off and loss of communication, a backup power module can be added to the power system to help robot auto return and recovery.

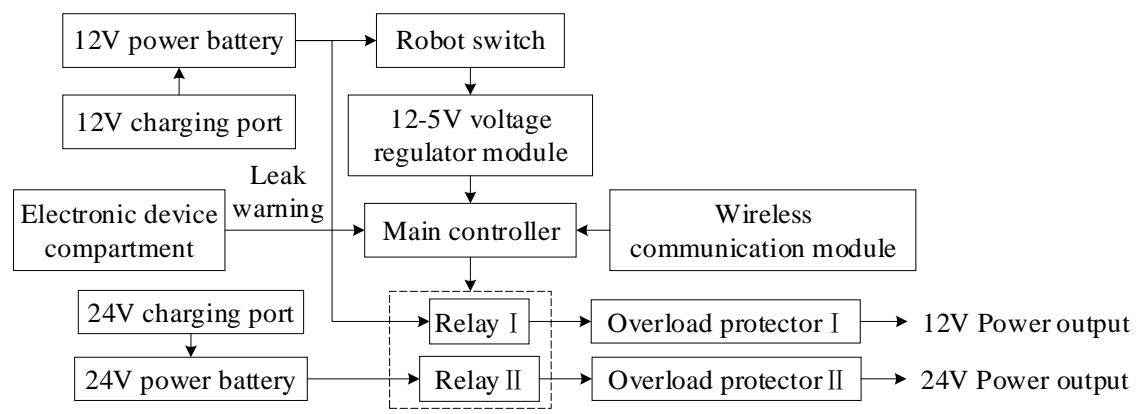

Figure 10. Working principle of the power module

\subsubsection{Motion control}

(1) Horizontal plane track control

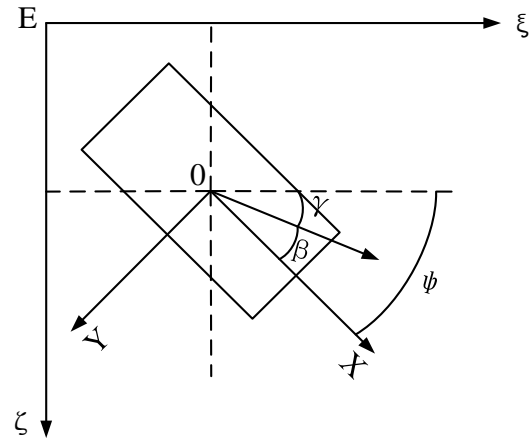

Figure 11. Robot motion in the horizontal plane
The horizontal plane motion model of the robot is shown in Figure 11. Point $\mathrm{E}$ is a certain position on the horizontal plane, $\xi$ points directly to the right ahead direction of the initial position of the robot, and $\zeta$ points to the downward direction, which is determined by the right-hand rule. The 0 point is the position of the center of gravity of the robot, the $X$ axis points to the right ahead direction of the robot, the $\mathrm{Z}$ axis is perpendicular to the water surface and points to the downward direction, and the $\mathrm{Y}$ axis is determined by the right-hand rule.

Assume the heading angle is $\psi$, the hydrodynamic angle is $\beta$, the robot's rotation angular velocity is $r$, and the horizontal plane track azimuth of the robot is $\gamma$, then there is:

$$
\left\{\begin{array}{l}
\dot{\psi}=\mathrm{r} \\
\gamma=\psi-\beta
\end{array}\right.
$$


The navigation module controls the positioning of robot so that it could accurately enters the predetermined plane and maintains the position, the positioning control loop is shown in Figure 12. In the figure, $\left(X_{i}, Y_{i}, Z_{i}\right)$ is the given value of the control system, $\left(X_{0}, Y_{0}, Z_{0}\right)$ is the actual value output by the navigation module. The navigation module consists of two parts, one part uses BDS module for surface navigation, and the other part is underwater and uses inertial navigation. This study adopted a typical PID regulator for positioning control. By controlling the forward and backward rotation of the propeller, the rotation speed of the motor, and the angle of the propeller in the vector thruster, the track azimuth $\gamma$ could be adjusted to achieve the thrust distribution of the three propellers. In order to move the robot carrier from a given point to a target point, after the robot dives to a specified depth, the vector propulsion device starts to work, the robot performs a yawing motion, and the track azimuth angle $\gamma$ changes accordingly, when $\gamma$ reaches the specified angle, the robot starts to move forward, after reaching the specified position, it stops moving.

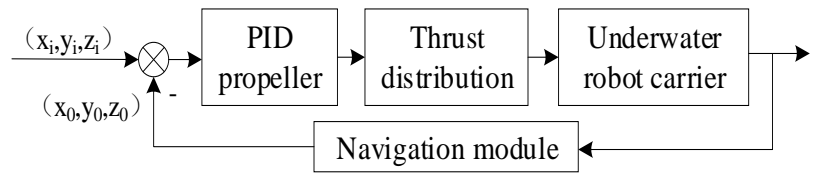

Figure 12. Positioning control loop

(2) Vertical plane depth control

The vertical plane motion model of the robot is shown in Figure 13. Point $\mathrm{E}$ is a certain position on the vertical plane, $\zeta$ points to the downward direction, $\xi$ points directly to the right ahead direction of the initial position of the robot, and $\zeta$ is determined by the right-hand rule.

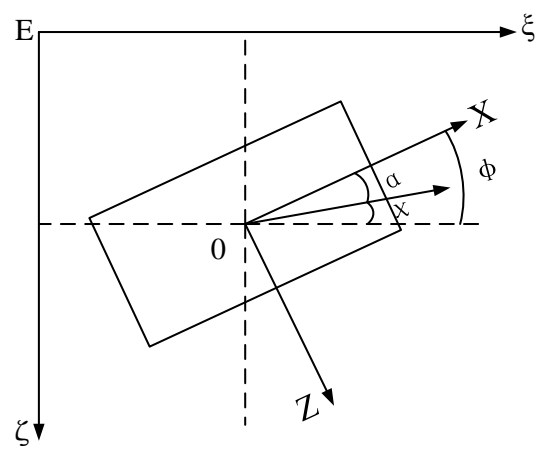

Figure 13. Vertical motion of the robot

Assume the pitch angel of the robot on the vertical plane is $\phi$, the track angel is $\chi$, the hydrodynamic angle is $\alpha$, the angular velocity of the robot's trimming motion is $q$, then there is:

$$
\left\{\begin{array}{l}
\dot{\phi}=q \\
\chi=\phi-\alpha
\end{array}\right.
$$

To achieve sailing at a given depth in the vertical plane, the robot must perform automatic depth keeping control. The robot adopts a three-propeller propulsion method, its depth keeping adopts the series control method, the outer loop is the depth control loop, and the inner loop is the pitch control loop. The depth keeping control system [22-28] of the robot converts the voltage signal fed back by the pressure transmitter into the depth signal, the depth control loop is shown in Figure 14. $D(t)$ is the current depth measured by the pressure transmitter, $\phi(t)$ is the current pitch angle of the robot. The input of the system is the target depth, and the fixed-force propeller controls the speed of the propeller according to the collected speed signal, so as to realize the depth keeping control of the robot.

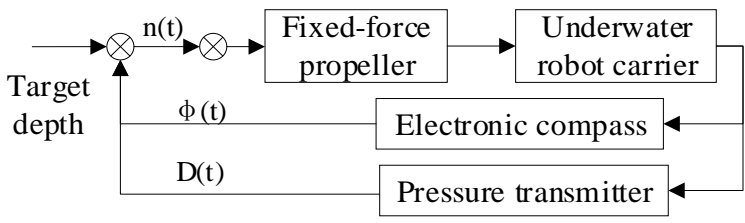

Figure 14. Depth control loop

\subsection{Remote monitoring terminal}

Users can install remote monitoring terminal on PC to establish the communication link between the main controller and the PC, and query the water quality information and the navigation status of the robot in real time. The remote monitoring terminal uses a wireless serial communication module (model ATK-LORA-01, manufactured by Guangzhou Xingyi electronic technology co. LTD, Guangzhou, Guangdong, China) to receive data sent back by the robot. The communication module has a communication distance of more than 3 kilometers and a wireless rate of $19200 \mathrm{bps}$, its antiinterference performance is good, and the communication is stable, which can achieve information transmission in shallow waters and meet the working requirements of the robot. $\mathrm{C}++$ language was used to develop the software of the remote monitoring terminal host computer. The software interface includes four modules: water quality information monitoring system, robot navigation monitoring and control system, communication control system and operation status monitoring system, as shown in Figure 15.

Users can directly read the detection data from the remote monitoring terminal to get the water quality information, the water quality detection information interface is shown in Figure 16. The remote monitoring terminal can also display the robot's navigation information in real time, so that users can learn about the robot's work conditions in time. The robot navigation information interface is shown in Figure 17.

The workflow of the robot is shown in Figure 18. When the robot starts to work, the system is initialized first, and the operator needs to set the position information of the water area to be detected; then the robot marches to the intended position, after reaching the specified position on the horizontal plane, the robot dives to the specified depth, performs water quality detection, stores the detection results and sends back to the remote monitoring terminal, which will display the data on the host computer in real time. After that, the robot floats to the surface of the water, travels to the next detection position, and repeats the operation steps. In this process, the main control program controls the robot to dive and reach the specified depth with the help of the depth keeping control system, then the program is stopped to process the data obtained by the sensors on the robot, and collect parameters such as depth, water temperature, $\mathrm{pH}$, and $\mathrm{DO}$, etc. 


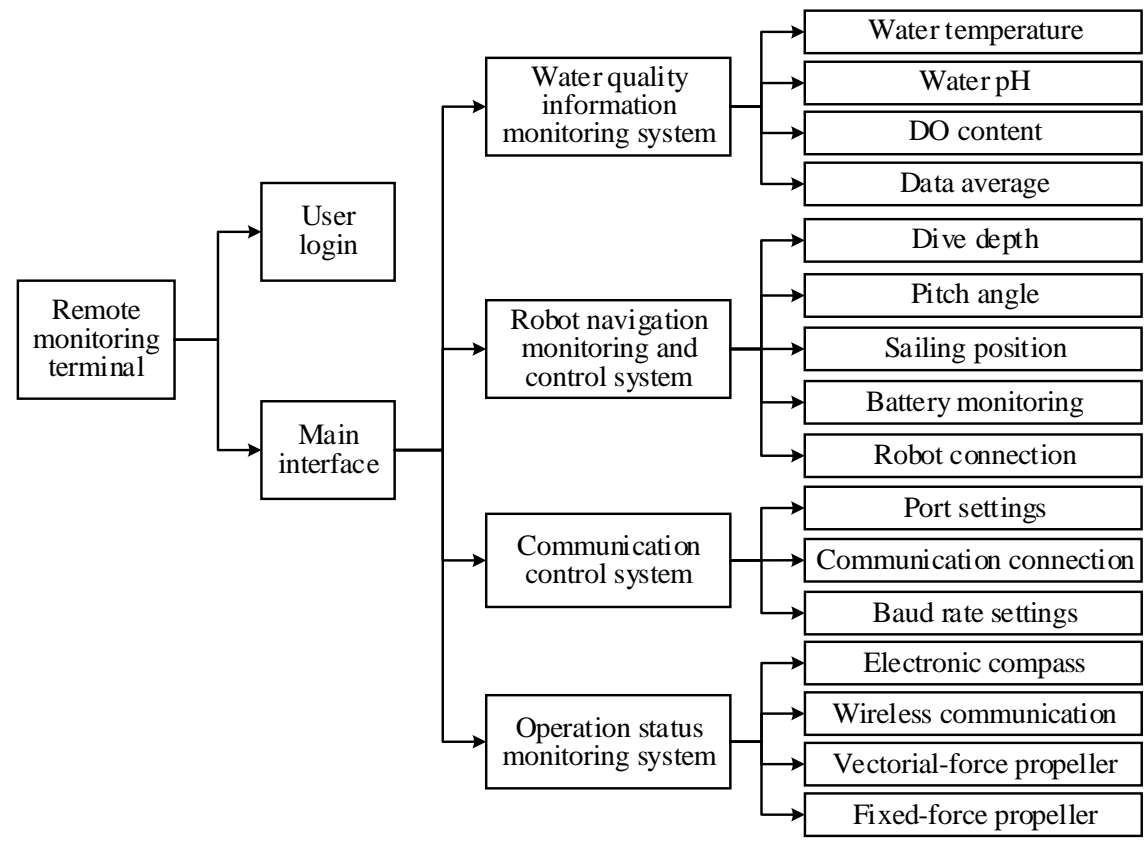

Figure 15. Program structure of remote monitoring terminal

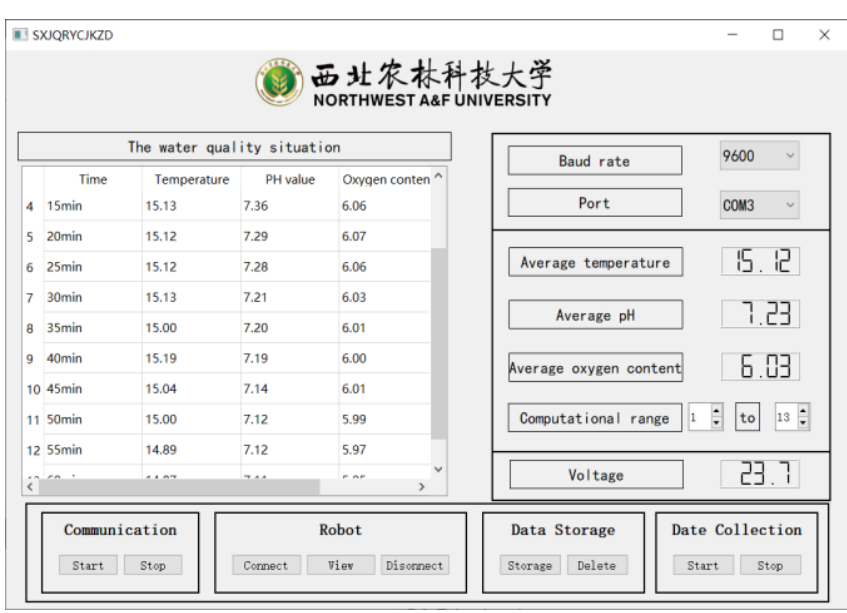

Figure 16. Water quality detection information interface of the remote monitoring terminal

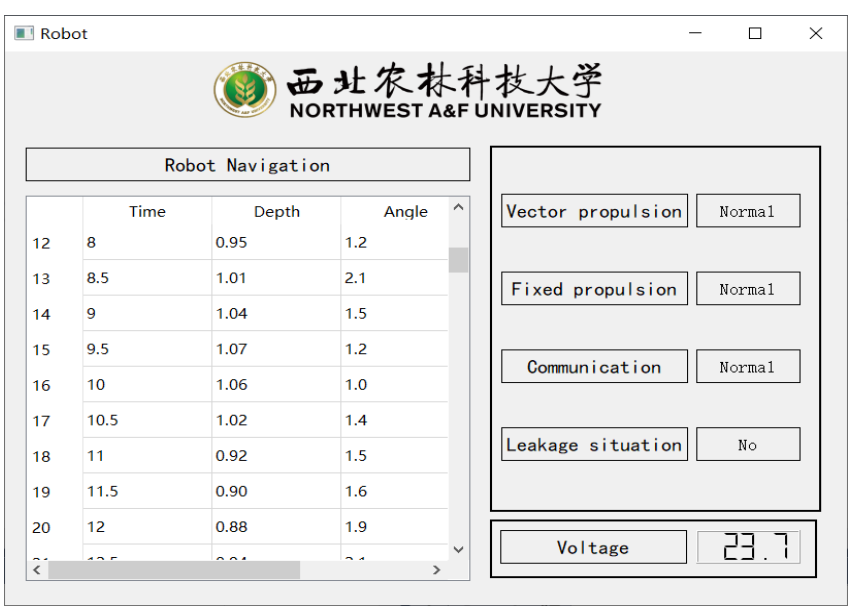

Figure 17. Robot parameter interface of the remote monitoring terminal

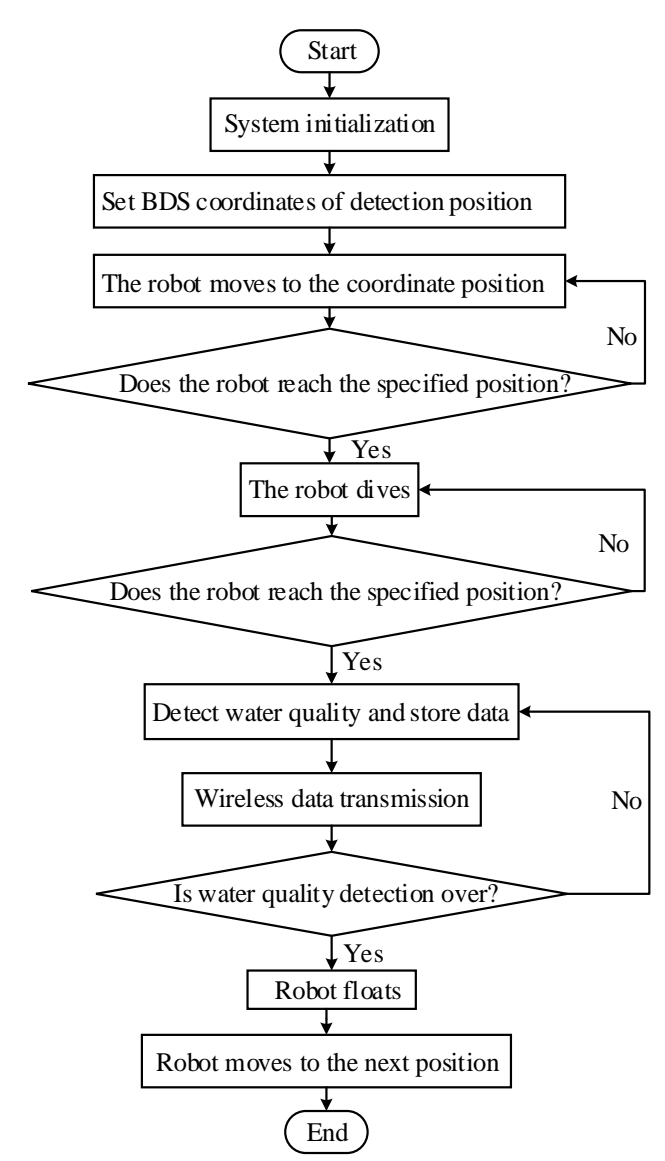

Figure 18. Robot workflow

\section{TEST AND CONCLUSION}

To test the reliability of the robot system, this study conducted navigation test, diving test and water quality test to verify the robot's horizontal plane navigation control performance, vertical plane navigation control performance, 
dive depth control performance, and the data acquisition performance of the water quality detection devices.

\subsection{Navigation test}

The navigation performance of the robot is a guarantee to realize other functions of the robot system, so the navigation test occupies an important position in the tests for the water quality detection robots, and it is also a test for the reliability of the entire control system. In order to verify the reliability of the control system when the robot is sailing on different planes, both horizontal plane sailing test and vertical plane sailing test were performed, and the test environment is shown in Figure 19. The specific test site is a circular pool located in front of the north campus library of the Northwest A \& F University, the pool has an outer ring diameter of $24 \mathrm{~m}$ and an inner ring diameter of $20 \mathrm{~m}$, and its water depth is $1.5 \mathrm{~m}$.

To verify the reliability of the control system when the robot is sailing on horizontal plane, the horizontal plane sailing test was conducted. First, the course of the robot was preset to be an arc, then the robot was placed in the pool and kept in a floating state, and the remote monitoring terminal was used to control the robot to start sailing. In the test process, the actual sailing trajectory of the robot was recorded and compared with the preset course trajectory, the results are shown in Figure 20.

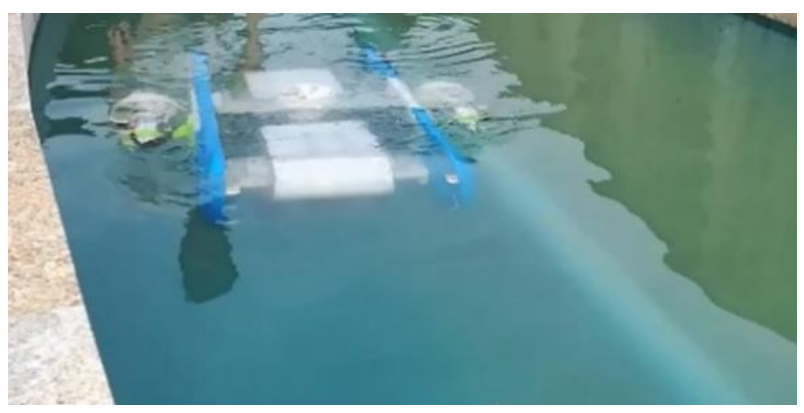

Figure 19. Test scene of the water quality detection robot in the pool

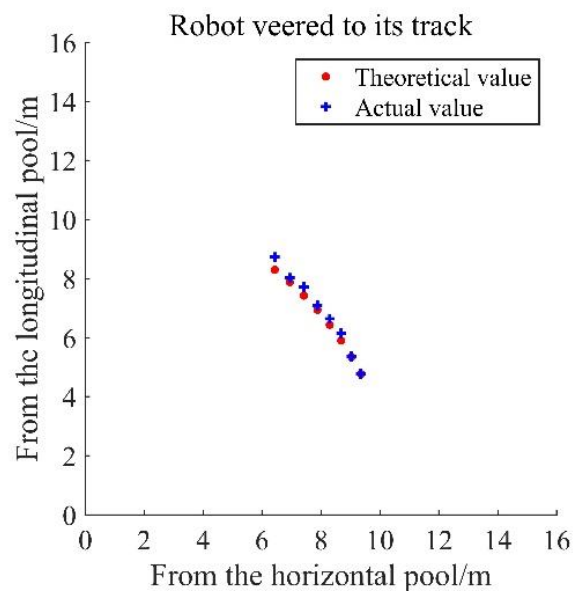

Figure 20. Comparison of sailing trajectories

It can be seen from the figure that the actual trajectory and the preset trajectory basically matched with each other, the maximum motion error was less than $0.45 \mathrm{~m}$, which had met the accuracy requirement of the navigation control. Therefore, the robot can follow the preset path on the horizontal plane, and the control system has a high reliability.
To verify the reliability of the control system when the robot is sailing on vertical plane, the vertical plane sailing test was conducted. The vertical initial position of the robot was set at $1.5 \mathrm{~m}$ and the horizontal initial position was set at $1 \mathrm{~m}$. Then the robot was placed in the pool and kept in a floating state, and the remote monitoring terminal sent orders to start the fixedforce propeller, so as to drive the robot to dive, and the position information of the robot during the diving process was recorded. The recorded depth and trajectory data were compared with the preset data, and the comparison results are shown in Figure 21.

It can be seen from the figure that, under the condition of slow sailing speed, the robot's dive motion control deviation did not exceed $0.15 \mathrm{~m}$, the robot's dive control performance was good, but the longitudinal offset error was relatively large. This is because the defect in the design and processing of the casing would cause uneven resistance, plus the impact of underwater disturbance forces during the robot diving process, so it had resulted in a larger deviation in the navigation on the vertical plane. To reduce the error in the longitudinal diving motion, the vectorial-force propellers installed on both sides could be used to assist balancing, so as to meet the requirements of the straight-line sailing motion on the vertical plane.

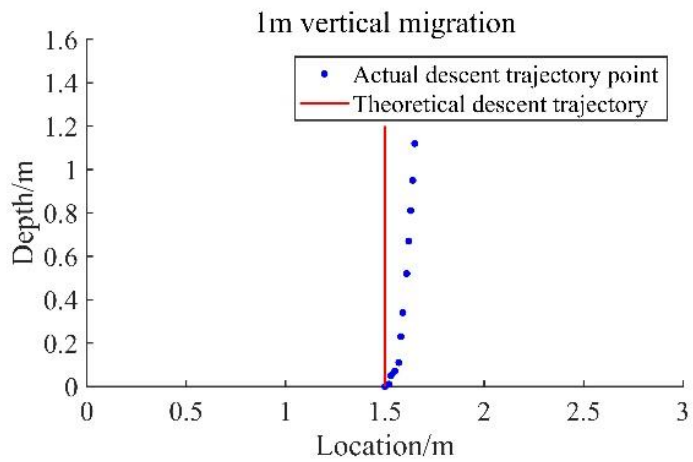

(a) Vertical offset curve

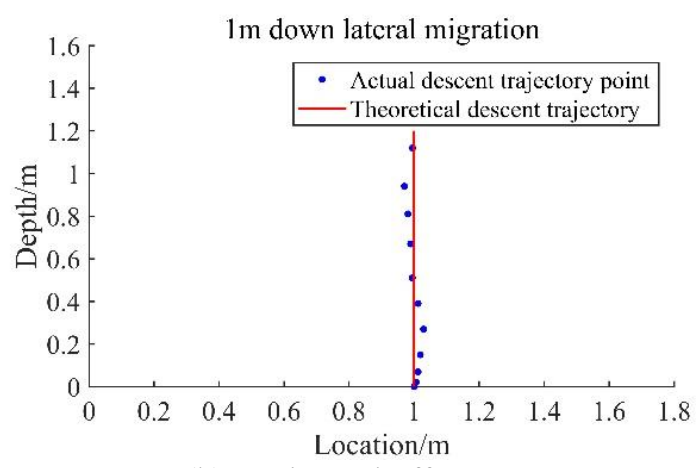

(b) Horizontal offset curve

Figure 21. Robot diving and floating trajectories

\subsection{Diving test}

To further verify the feasibility of normal operation of the robot at specified water depth, the diving test was performed in the Weihe River water area in Yangling District, Shaanxi Province, China. In the test, the target dive depth of the robot was set to 5 meters, then the robot was placed in the river and kept in a floating state, then the remote monitoring terminal sent orders to control the robot to dive, and the depth information of the robot during the diving process was 
recorded, and the dive depth curve was plotted as shown in Figure 22 .

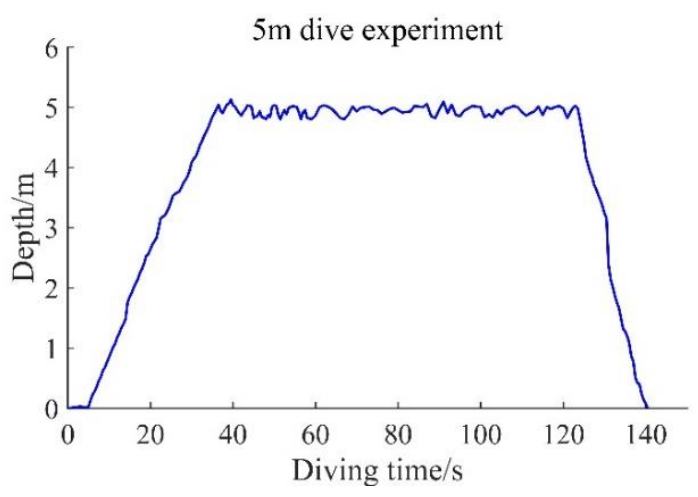

Figure 22. Curve of the dive depth of the robot

In the diving process, according to the depth information and target depth information fed back by the pressure transmitter and the angle information fed back by the electronic compass, the depth keeping control system made comparisons and controlled the allocation of the thruster of propellers, so that the robot dived to the target depth. From the figure we can see that, the robot's dive time was about 38 s, and it remained basically stable at the target depth position. Compared with the target depth, the depth deviation of the stabilized robot was within $\pm 0.20 \mathrm{~m}$, so the robot had a good depth keeping control performance, which met the working requirements.

\subsection{Water quality detection test}

In this test, the dive depth of the robot was set to 2 meters, and the robot was placed in the pool and kept in a floating state, then the remote monitoring terminal sent orders to control the robot to start working, the robot collected the three parameters of the water temperature, $\mathrm{pH}$ and $\mathrm{DO}$ within 1 hour from the detection points in the pool, and the changes of the three water quality indicators over time $t$ within 1 hour are shown in Figure 23.
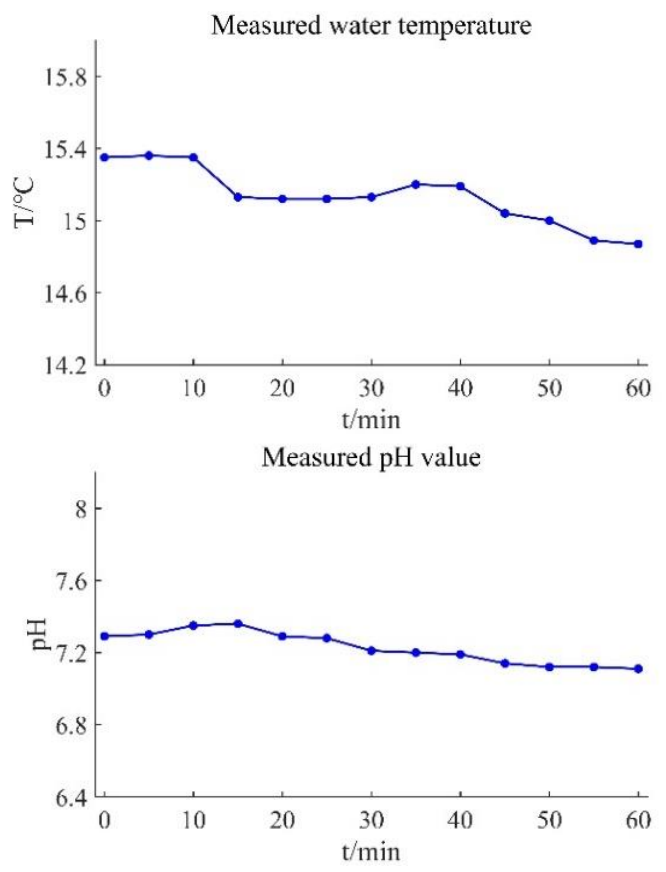

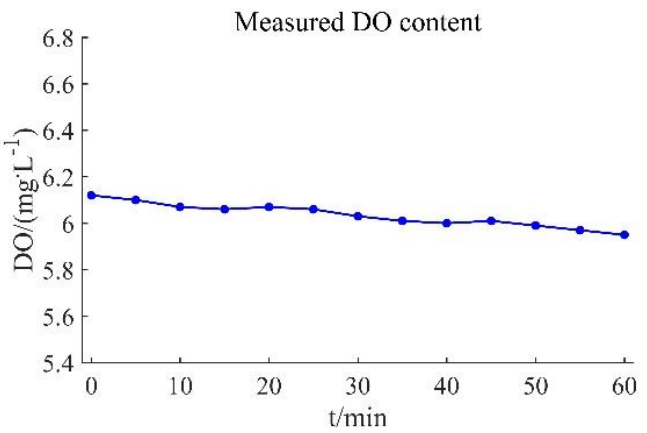

Figure 23. Changes of measured water quality indicators over time within $1 \mathrm{~h}$

The robot could detect the three water quality indicators at the same time, for every test point, the robot only needed less than $5 \mathrm{~min}$ to finish the detection; by comprehensively analyzing the overall trend of the test results we can see that, using underwater robot to perform real-time water quality detection is of certain feasibility, the method is time-saving, and sampling is not required in this method. Therefore, the method of using underwater robot to carry water quality detection devices is simple in operation, and it can complete the water quality detection tasks without the need of sampling.

\section{CONCLUSION}

This paper analyzed the environment requirements of freshwater aquaculture and designed a water quality detection robot. The robot used two propellers to perform vectorial-force propulsion, one propeller to perform fixed-force propulsion, so as to adapt to the shallow water environment and perform autonomous movements. A prototype was subject to relevant tests, the sensor data were analyzed, and the test results showed that, the robot's navigation control and dive depth control can ensure the accuracy of the movement and meet the working requirements, wherein the vertical plane motion error was relatively large, but the vectorial-force propellers could be used to assist balancing, so as to meet the accuracy requirements of the motion control. Compared with the traditional water quality detection robots, the proposed robot is flexible in motions, the equipped water quality detection module could collect water parameters such as temperature, $\mathrm{pH}$ and $\mathrm{DO}$, achieving water quality detection of the breeding environment. The robot integrated various functions such as early warning and data storage, it has multiple functions, low cost, and high reliability. On the one hand, the robot can greatly increase the level of informatization, automation, and intelligence of the aquaculture industry, which is conducive to reducing costs, improving productivity and management efficiency; on the other hand, it can provide a reference for further expanding the application range of agricultural information collection devices, so it has a broad market in the field of hydrological environment detection.

\section{ACKNOWLEDGMENT}

This research has received support from the Shaanxi Key Research and Development Program of China (2019NY-171, 2019ZDLNY02-04, 2018TSCXL-NY-05-04). The authors are 
also gratefully to the reviewers for their helpful comments and recommendations, which make the presentation better.

\section{REFERENCES}

[1] Duan, Y., Li, X., Zhang, L., Chen, D., Ji, H. (2020). Mapping national-scale aquaculture ponds based on the Google Earth Engine in the Chinese coastal zone. Aquaculture, 520: 734666. https://doi.org/10.1016/j.aquaculture.2019.734666

[2] Purcell, S.W., Williamson, D.H., Ngaluafe, P. (2018). Chinese market prices of beche-de-mer: Implications for fisheries and aquaculture. Marine Policy, 91: 58-65. https://doi.org/10.1016/j.marpol.2018.02.005

[3] Zhang, T., Li, Q., Zhang, C.S., Liang, H.W., Li, P., Wang, T.M., Li, S., Zhu, Y.L., Wu, C. (2017). Current trends in the development of intelligent unmanned autonomous systems. Frontiers of Information Technology \& Electronic Engineering, 18(1): 68-85. https://doi.org/10.1631/FITEE.1601650

[4] Duan, Q., Liu, Y., Zhang, L., Li, D. (2018). State-of-theart review for application of big data technology in aquaculture. Transactions of the Chinese Society for Agricultural Machinery, 49(6): 1-16.

[5] Fore, M., Frank, K., Norton, T., Svendsen, E., Alfredsen, J.A., Dempster, T., Eguiraun, H., Watson, W., Stahl, A., Sunde, L.M., Schellewald, C., Skøien, K.R., Alver, M.O., Berckmans, D. (2017). Precision fish farming: A new framework to improve production in aquaculture. Biosystems Engineering, 173: 176-193. https://doi.org/10.1016/j.biosystemseng.2017.10.014

[6] Han, Q.X., Keesing, J.K., Liu, D. (2016). A review of sea cucumber aquaculture, ranching, and stock enhancement in China. Reviews in Fisheries Science \& Aquaculture, 24(4): https://doi.org/10.1080/23308249.2016.1193472

[7] Huang, J., Meng, X., Xie, Q., Chen, W., Wang, S. (2017). Complete Sets of Aquaculture Automation Equipment and Their Monitoring Cloud Platform. International Conference on Mechatronics, pp. 429-435. https://doi.org/10.1007/978-3-319-70990-1_63

[8] Anikuttan, K.K., Adhikari, S., Kavitha, M., Jayasankar, P. (2016). Carbon sequestration capacity of sediments, algae, and zooplankton from fresh water aquaculture ponds. Environmental Monitoring and Assessment, 188(7): 422-422. https://doi.org/10.1007/s10661-0165430-0

[9] Ma, C.G., Zhao, D.A., Wang, J.G., Chen, Y.J., Li, Y.Z. (2015). Intelligent monitoring system for aquaculture dissolved oxygen in pond based on wireless sensor network. Transactions of the Chinese Society of Agricultural Engineering, 31(7): 193-200.

[10] Parra, L., Sendra, S., García, L., Lloret, J. (2018). Design and deployment of low-cost sensors for monitoring the water quality and fish behavior in aquaculture tanks during the feeding process. Sensors, 18(3): 750 https://doi.org/10.3390/s18030750

[11] Hong, J., Zhao, D., Sun, Y, Zhang, J., Luo, J. (2017). Multi model adaptive control of paddlewheel vehicle's course in aquaculture. Transactions of the Chinese Society of Agricultural Engineering, 33(1): 95-101. https://doi.org/10.11975/j.issn.1002-6819.2017.01.013

[12] Li, D.L., Bao, J.H. (2018). Research progress on key technologies of underwater operation robot for aquaculture. Transactions of the Chinese Society of Agricultural Engineering.

[13] Li, Z., Wang, J., Li, D. (2016). Applications of Raman spectroscopy in detection of water quality. Applied Spectroscopy Reviews, 51(4): 333-357. https://doi.org/10.1080/05704928.2015.1131711

[14] Salim, T.I., Haiyunnisa, T., Alam, H.S. (2016). Design and implementation of water quality monitoring for eel fish aquaculture. 2016 International Symposium on Electronics and Smart Devices (ISESD). https://doi.org/10.1109/ISESD.2016.7886720.

[15] Li, X.X., Zhu, C.G., Zhou, J., Sun, L.Q., Cao, X.M., Zhang, X.S. (2018). Review and trend of water quality detection in aquaculture by spectroscopy technique. Transactions of the Chinese Society of Agricultural Engineering, 34(19): 184-194. https://doi.org/10.11975/j.issn.1002-6819.2018.19.024

[16] Cheng, X.J., Ju, J.Q., Hu, J.C., Xie, J., Yu, D.G. (2019). Response model construction of fish growth to water environmental factors in recirculating aquaculture mode. Transactions of the Chinese Society of Agricultural Engineering (Transactions of the CSAE), 35(11): 188194. https://doi.org/10.11975/j.issn.10026819.2019.11.022

[17] Zhou, C., Zhang, B., Lin, K., Xu, D., Chen, C., Yang, X., Sun, C. (2017). Near-infrared imaging to quantify the feeding behavior of fish in aquaculture. Computers and Electronics in Agriculture, 135: 233-241. https://doi.org/10.1016/j.compag.2017.02.013

[18] Wu, T., Huang, Y., Chen, J. (2015). Development of an adaptive neural-based fuzzy inference system for feeding decision-making assessment in silver perch (Bidyanus bidyanus) culture. Aquacultural Engineering, 66: 41-51. https://doi.org/10.1016/j.aquaeng.2015.02.001

[19] Li, Y., Jiang, Y., Wang, L., Cao, J., Zhang, G. (2015). Intelligent PID guidance control for AUV path tracking. Journal of Central South University, 22(9): 3440-3449. https://doi.org/10.1007/s11771-015-2884-0

[20] Gao, F., Han, Y., Wang, H., Ji, G. (2017). Innovative design and motion mechanism analysis for a multimoving state autonomous underwater vehicles. Journal of Central South University, 24(5): 1133-1143. https://doi.org/10.1007/s11771-017-3516-7

[21] Song, Y.S., Arshad, M.R. (2017). Robust optimal depth control of Hovering Autonomous Underwater Vehicle. 2017 IEEE 2nd International Conference on Automatic Control and Intelligent Systems (I2CACIS). 191-195. https://doi.org/10.1109/i2cacis.2017.8239056

[22] Zeng, J.B., Li, S., Li, Y.P., Wang, X.H., Yan, S.X. (2016) Research and application of the control system for a portable autonomous underwater vehicle. Robot, 38(1): 91-97.

[23] Mirzaei, M., Taghvaei, H. (2019). A Full Hydrodynamic Consideration in Control System Performance Analysis for an Autonomous Underwater Vehicle. Journal of Intelligent and Robotic Systems, 1-17. https://doi.org/10.1007/s10846-019-01102-1

[24] Li, Y., Guo, S., Wang, Y. (2017). Design and characteristics evaluation of a novel spherical underwater robot. Robotics and Autonomous Systems, 94: 61-74. https://doi.org/10.1016/j.robot.2017.03.014

[25] Guo, J., Wu, G., Guo, S. (2015). Fuzzy PID algorithmbased motion control for the spherical amphibious robot. 
122

International Conference on Mechatronics and Automation, pp. 1583-1588. https://doi.org/10.1109/ICMA.2015.7237721

[26] Tumari, M.Z., Abidin, A.F., Hussin, M.S., Kadir, A.M., Aras, M.S., Ahmad, M.A. (2019). PSO fine-tuned model-free PID controller with derivative filter for depth control of hovering autonomous underwater vehicle. Methods of Molecular Biology, 3-13. https://doi.org/10.1007/978-981-13-3708-6_1
[27] Li, Y., Guo, S., Yue, C. (2015). Preliminary concept of a novel spherical underwater robot. International Journal of Mechatronics and Automation, 5(1): 11-21. https://doi.org/10.1504/IJMA.2015.068449

[28] Wu, Y., Ta, X., Xiao, R., Wei, Y., An, D., Li, D. (2019). Survey of underwater robot positioning navigation. Applied Ocean Research, 90: 101845. https://doi.org/10.1016/j.apor.2019.06.002 